\title{
Inhibition of NOX2 reduces locomotor impairment, inflammation, and oxidative stress after spinal cord injury
}

\author{
Guzal Khayrullina, Sara Bermudez and Kimberly R. Byrnes*
}

\begin{abstract}
Background: Spinal cord injury $(\mathrm{SCl})$ results in the activation of the NADPH oxidase (NOX) enzyme, inducing production of reactive oxygen species (ROS). We hypothesized that the NOX2 isoform plays an integral role in post-SCl inflammation and functional deficits.

Methods: Moderate spinal cord contusion injury was performed in adult male mice, and flow cytometry, western blot, and immunohistochemistry were used to assess NOX2 activity and expression, inflammation, and M1/M2 microglia/macrophage polarization from 1 to 28 days after injury. The NOX2-specific inhibitor, gp91ds-tat, was injected into the intrathecal space immediately after impact. The Basso Mouse Scale (BMS) was used to assess locomotor function at $24 \mathrm{~h}$ post-injury and weekly thereafter.
\end{abstract}

Results: Our findings show that gp91ds-tat treatment significantly improved functional recovery through 28 days post-injury and reduced inflammatory cell concentrations in the injured spinal cord at $24 \mathrm{~h}$ and 7 days post-injury. In addition, a number of oxidative stress markers were reduced in expression at $24 \mathrm{~h}$ after gp91ds-tat treatment, which was accompanied by a reduction in M1 polarization marker expression.

Conclusion: Based on our findings, we now conclude that inhibition of NOX2 significantly improves outcome after $\mathrm{SCl}$, most likely via acute reductions in oxidative stress and inflammation. NOX2 inhibition may therefore have true potential as a therapy after SCl.

Keywords: NOX2, Polarization, Microglia, Inflammation, Spinal cord injury, Oxidative stress

\section{Background}

Spinal cord injury (SCI) is followed by an acute but long-lasting inflammatory response, marked by invasion of blood-borne cells and activation of endogenous cells and a marked increase in reactive oxygen species (ROS) production. Neutrophils are the first cells to respond, invading the spinal cord within minutes to hours and populating the injured tissue for days [1]. Following this response is a macrophage/microglial response that typically peaks within 4-7 days [2]. Both of these cell types express the NADPH oxidase (NOX) 2 enzyme, which is a primary producer of ROS in the spinal cord after injury.

\footnotetext{
* Correspondence: kimberly.byrnes@usuhs.edu

Anatomy, Physiology and Genetics Department, Uniformed Services University, Room B2048, 4301 Jones Bridge Road, Bethesda, MD 20814, USA
}

Research has shown that this macrophage/microglia response is not homogenous, and both cell types can be polarized into pro-inflammatory M1 cells or antiinflammatory M2 cells [3]. Early work demonstrated that, after SCI, the M1/M2 ratio is roughly equal acutely after injury but shifts to an M1 dominant response within a few days [4]. It is currently unclear what signals result in this M1/M2 shift, although some research has suggested that the NOX enzyme may play a role in not only this polarization [5] but also general inflammatory responses and oxidative stress.

The NOX enzyme complex is a primary source of ROS, with seven currently known family members, including NOX2 [6]. The enzyme functions by transferring electrons from NADPH inside the cell, across the membrane, and then to extracellular oxygen, generating a superoxide. Components of the NOX2 enzyme, including 
the enzymatic core, gp91 ${ }^{\mathrm{PHOX}}$, and membrane-bound companion protein, $\mathrm{p} 22^{\mathrm{PHOX}}$, are elevated after SCI [7-11], and NOX activity is upregulated for at least 6 months post-injury [9].

The NOX2-specific inhibitor, gp91ds-tat, a 20-amino acid peptide that interferes with the assembly of activated NOX2, has been used in research for a number of different disease states, both in vitro and in vivo designs. In vitro, gp91ds-tat effectively reduces the release of ROS from activated microglia [12]. Previously, we found that systemic administration of gp91ds-tat reduced both oxidative stress and cytokine production acutely after SCI [11]. In addition, gp91ds-tat was found to be neuroprotective in a traumatic brain injury model [13].

The aim of the current paper was to explore the effect of specific inhibition of NOX2 on oxidative stress, inflammation, and function after SCI. We now show that inhibition of NOX2 reduces post-injury inflammation and improves locomotor recovery in mice. In addition, we show that NOX2 inhibition shifts microglial/macrophage polarization toward the M2 phenotype, which may have significant neuroprotective effects in the injured spinal cord.

\section{Methods}

\section{Animal handling and surgical methods}

Adult male C57Bl6 mice were utilized in all experiments (20-25 g, Taconic Farms, Derwood, MD). Mice were group housed and received food and water ad libitum with a 12:12 h light cycle. A total of 111 male mice were used for this study; 13 mice were removed from the study due to post-surgical complications. All experiments complied fully with the principles set forth in the "Guide for the Care and Use of Laboratory Animals" and were approved by the Uniformed Services University IACUC.

All subjects undergoing surgery received isoflurane (Primal Healthcare, Andhra Pradesh, India). Mice received a laminectomy, followed by a contusion simulating moderate SCI using the Infinite Horizons Impactor (50 kdyn; Precision Systems and Instrumentation, Fairfax Station, VA). Mice were immediately given an intrathecal injection of either gp91ds-tat or scrambled ds-tat (AnaSpec, Inc., Fremont, CA) diluted to $50 \mu \mathrm{M}$ in saline in a $5 \mu$ l volume at the lesion epicenter. After expelling the liquid, the needle was held under the dura for $30 \mathrm{~s}$ prior to removal. The incision was then closed, and animals were maintained on heating pads until mice regained movement. Acetaminophen (Children's Tylenol, $200 \mathrm{mg} / \mathrm{kg}$ ) was added to drinking water for $72 \mathrm{~h}$ post-injury. Manual bladder expression was performed daily until normal bladder expression returned. Naïve mice did not undergo surgery or receive isoflurane.

\section{Functional testing}

The Basso Mouse Scale (BMS) was used to rate locomotor function and recovery. Injured mice in recovery were scored in seven categories including ankle movement, plantar placement, stepping, coordination, paw position, trunk instability, and tail position. Mice ( $n=9$ /group) from the 28-day time point group were observed at $24 \mathrm{~h}$ by two investigators blinded to the treatment group and then weekly post-injury.

\section{Immunohistochemistry}

At $2 \mathrm{~h}$ ( $n=4$ gp91ds-tat, 3 scrambled ds-tat), $24 \mathrm{~h}(n=4$ gp91ds-tat, 4 scrambled ds-tat), 7 days ( $n=3$ gp91ds-tat, 4 scrambled ds-tat), and 28 days ( $n=4$ gp91ds-tat, 4 scrambled ds-tat), injured or naïve $(n=4)$ mice were anesthetized (Euthasol, $0.22 \mathrm{ml} / \mathrm{kg}$, IP) and perfused with $100 \mathrm{ml}$ of $0.9 \%$ sterile saline, followed by $300 \mathrm{ml}$ of $10 \%$ buffered formalin phosphate (Fisher Scientific, Fair Lawn, NJ). A 5$\mathrm{mm}$ spinal cord segment, $2.5 \mathrm{~mm}$ caudal and $2.5 \mathrm{~mm}$ rostral to the injury site, was extracted. Spinal cords were kept in formalin for $24 \mathrm{~h}$ and then transferred to a $30 \%$ sucrose solution. Spinal cords were then cut into $20-\mu \mathrm{m}$ axial sections. Standard fluorescent immunohistochemistry was performed with primary antibodies that had been previously characterized in the laboratory $[11,12]$, including Iba1 (1:100, Wako), CD86 (1:200, Abcam), Mannose Receptor/CD206 (1:50, Abcam), and 3NT (1:5, Abcam). Alexa-Fluor secondary antibodies (Invitrogen) were used for visualization. Slides were coverslipped using mounting media containing DAPI to counterstain for nuclei (Vector Labs, Burlingame, CA).

Immunofluorescence was detected and photographed in the dorsal column region within the $5-\mathrm{mm}$ region of interest using an Olympus DP72 microscope with Olympus cellSens microscopy software (Olympus, Center Valley, PA) or NanoZoomer Digital Pathology system (Hamamatsu Photonics, K.K., Japan). For 3NT immunohistochemistry, fluorescent immunohistochemistry was quantified as previously described using pixel density measurement in Scion Image [14]. For all other immunohistochemistries, images were qualitatively evaluated for comparison to flow cytometry and western blotting results. For all stains, at least five sections taken from regular intervals within the 5 - $\mathrm{mm}$ region of interest were evaluated.

\section{Western blot}

At $24 \mathrm{~h}(n=4$ gp91ds-tat, 4 scrambled ds-tat), 7 days ( $n$ $=6$ gp91ds-tat, 5 scrambled ds-tat), and 28 days $(n=3$ gp91ds-tat, 3 scrambled ds-tat) post-injury or after no intervention (naïve, $n=8$ ), mice were euthanized and tissue was flushed with $100 \mathrm{ml}$ of $0.9 \%$ sodium chloride. A 5 -mm spinal cord segment, $2.5 \mathrm{~mm}$ rostral and $2.5 \mathrm{~mm}$ caudal to the lesion epicenter, was collected and protein 
extracted with RIPA (1x) buffer (Thermo Scientific, Rockford, IL). Aliquots of $25 \mu \mathrm{g}$ were used for western analysis using the following primary antibodies: CD86 (Abcam, 1:250), CD206 (Abcam, 1:500), iNOS (Cell Signaling Technology, 1:200), and phospho-p47 $7^{\mathrm{PHOX}}$ (p-p47; Sigma, 1:250). Immune complexes were detected with appropriate secondary antibodies and chemiluminescence reagents (Pierce, Rockford, IL). GAPDH was used as a control for gel loading and protein transfer. ImageJ software was used to quantify bands.

\section{Oxyblot}

All samples from the western blot experiments were also used for oxyblot experiments. Millipore OxyBlot Protein Oxidation Detection Kit (Temecula, CA) was used according to the manufacturer's instructions. $\beta$ actin (Abcam) was used as control for gel loading and protein transfer. ImageJ software was used to quantify resultant bands.

\section{Flow cytometry}

A 5-mm spinal cord segment, $2.5 \mathrm{~mm}$ caudal and $2.5 \mathrm{~mm}$ rostral to the injury site, was processed for flow cytometry at $24 \mathrm{~h}(n=4$ gp91ds-tat, 4 scrambled ds-tat $)$ and 7 days ( $n=8$ gp91ds-tat, 9 scrambled ds-tat) post-injury, following a perfusion with $100 \mathrm{ml}$ of $0.9 \%$ saline. Cells were isolated using a 70, 37, 30 \% Optiprep gradient (AxisShield, Wesbury, NY) in $1 \times$ HBSS solution. Cells were blocked with TruStain fcX (1:200 BioLegend, San Diego, $\mathrm{CA}$ ), and macrophages, microglia, lymphocytes, neutrophils, and dendritic cell populations were immunolabled with PE CD11b (1:200 eBioscience Inc., San Diego, CA), APC/Cy7 CD45(1:500, BioLegend, San Diego, CA), APC CD3e (1:200, BioLegend), and FITC GR1(1:2000 BioLegend, San Diego, CA) in flow cytometry staining buffer. Sytox Blue was used as a dead cell marker (1:1000 Molecular Probes, Inc. Eugene, OR).
OneComp eBeads (one drop per sample, eBioscience Inc., San Diego, CA) were used in place of tissue as marker controls, while tissue was used for unstained control for analysis purposes. Corresponding isotype controls were used per fluorochrome. Populations of interest were gated on, and at least 200,000 cells were collected for each sample. Data was analyzed using FlowJo software (FlowJo, LLC, Ashland, OR).

\section{Statistics}

Quantitative data are presented as mean \pm standard error of the mean. BMS and BMS subscore data were obtained by two blinded investigators and analyzed using repeated measures ANOVA with Bonferroni's multiple comparisons test. All other quantitative data were analyzed using unpaired $t$ test or one-way ANOVA as appropriate. All statistical tests were performed using the GraphPad Prism Program, Version 6.03 for Windows (GraphPad Software, San Diego, CA). A $p$ value $<0.05$ was considered statistically significant.

\section{Results}

\section{NOX2 inhibition improves locomotor function}

The BMS score provides a general locomotion score reflecting all four limbs, while the subscore reflects more distinct measures of locomotor control, such as plantar stepping, coordination, and paw position. Although both animal groups exhibited a gradual recovery, mice treated with gp91ds-tat showed an overall improvement with a significant improvement at 14- and 28-day time points compared to those treated with scrambled ds-tat (Fig. 1a). Furthermore, gp91ds-tat-treated mice demonstrated a recovery in more fine motor control, as shown in the BMS subscore, after 7 days with a significant improvement at 14 days (Fig. 1b). Scrambled ds-tat-treated mice only demonstrated an increase in BMS subscore at 28 days post-injury.

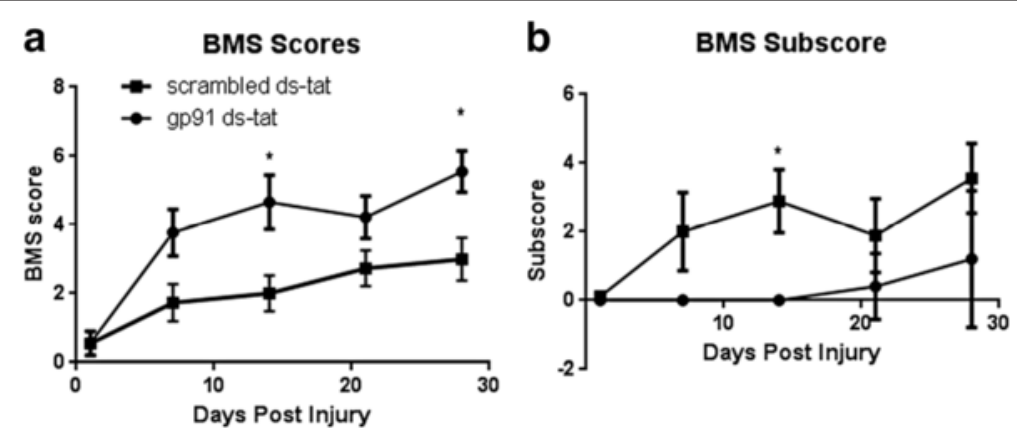

Fig. 1 Inhibition of NOX2 improves motor function. Gross hindlimb motor function was scored using the BMS (a), with further analysis of hindpaw function with the BMS subscore (b). Mice were tested at days 1, 7, 14, 21, and 28 post-injury. Those treated with gp91ds-tat showed significant increases in BMS score and BMS subscore at 7 days; this improvement was sustained through 28 days in the BMS score. Points represent mean \pm SEM. $N=9 /$ group. ${ }^{*} p<0.05$, repeated measures ANOVA 


\section{NOX2 inhibition reduces acute oxidative stress}

An oxyblot assay, which detects carbonylated proteins, was performed to measure the overall oxidative stress present in the injured tissue (Fig. 2a). While injury resulted in an increase in carbonylated proteins at $24 \mathrm{~h}$, carbonylation of proteins was significantly reduced in tissue that received gp91ds-tat in comparison to the scrambled ds-tat (Fig. 2b).

To further investigate oxidative stress, tissue was stained with an antibody against 3NT, a marker for nitrotyrosine- containing proteins (Fig. 2c, d). At $24 \mathrm{~h}$ post-injury, scrambled ds-tat-treated tissue demonstrated elevated $3 \mathrm{NT}$ immunostaining in both white and gray matters in comparison to naïve tissue. Qualitatively, gp91ds-tattreated spinal cord showed less staining at both $24 \mathrm{~h}$ and 7 days post-injury. At 28 days post-injury, residual expression in the lesion site was still observed in both groups, although reduced in comparison to earlier time points. When the immunostain was quantified, no significant differences were observed between the gp91ds-tat and a

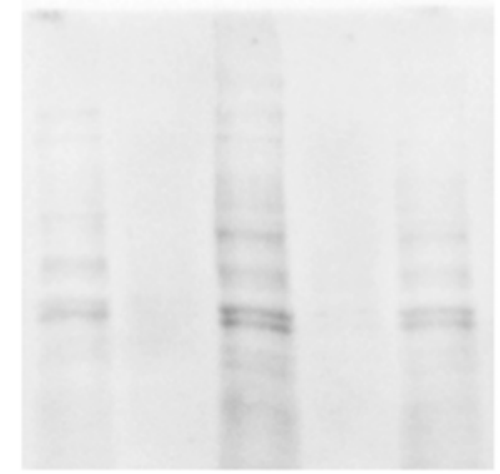

\section{Naïve Scrambled gp91}

d
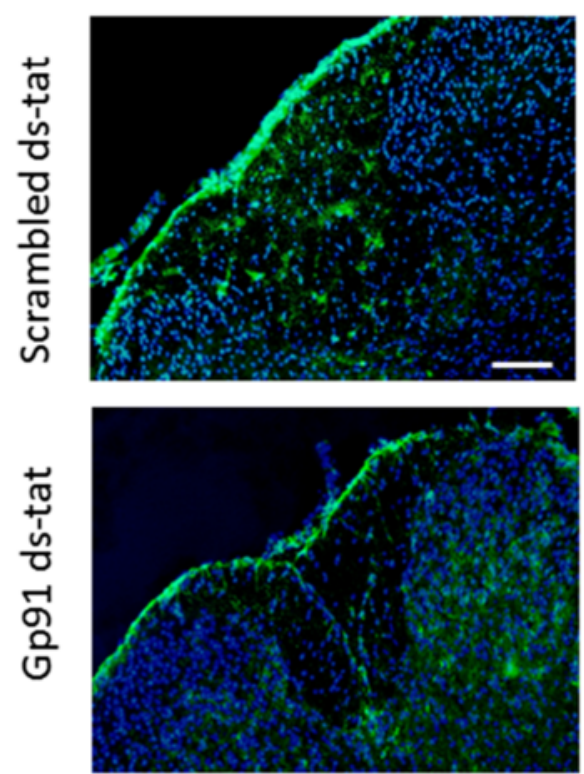

b

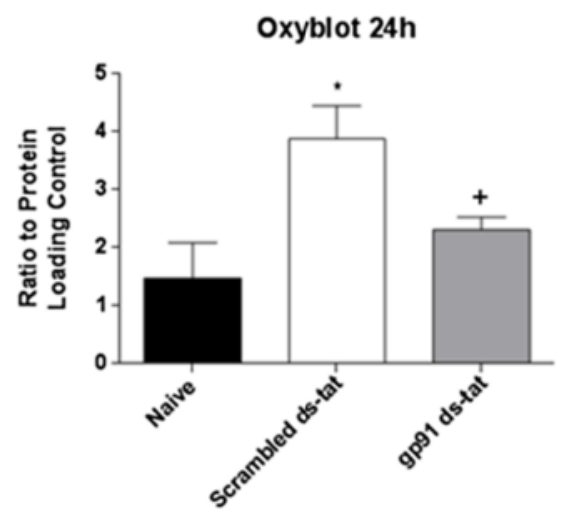

$7 \mathrm{DPI}$
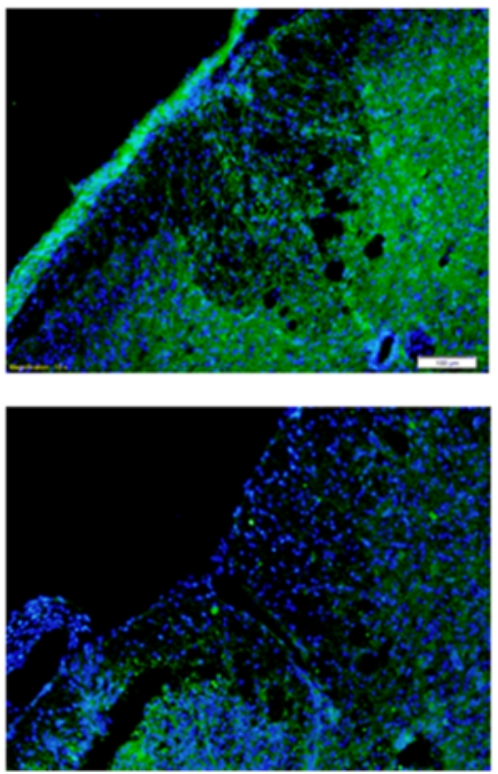

C
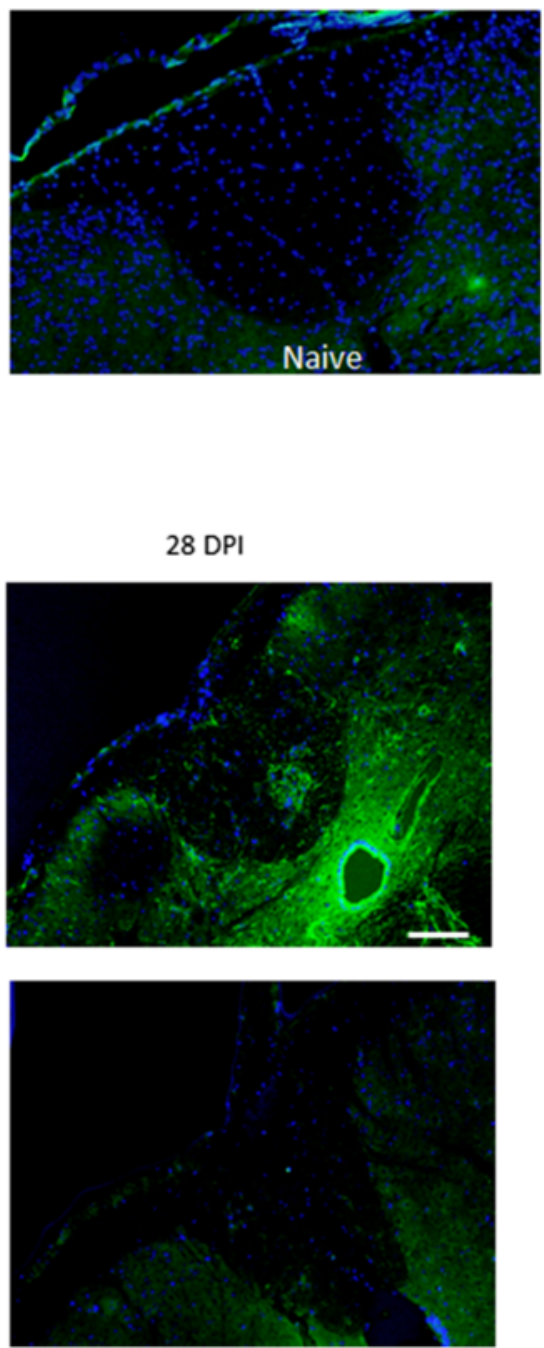

Fig. 2 Acute inhibition of NOX2 reduces markers of oxidative stress. OxyBlot Protein Oxidation assay was used to detect protein carbonylation in naïve $(n=8)$, scrambled ds-tat- $(n=4)$, and gp91ds-tat-treated mice $(n=4)$ at $24 \mathrm{~h}$ post-injury (a). Densitometry demonstrated a significant increase in protein carbonylation in scrambled ds-tat-treated mice, which was significantly reduced to naïve levels with gp91ds-tat treatment (b). At these same time points, spinal cord tissue was immunolabeled for 3-nitrotyrosine (green), a marker of nitrosylated protein (scrambled ds-tat: $n=4 /$ time point; gp91ds-tat: $n=4 / 24$ h, 3/7 days; 4/28 days). DAPI nuclear stain is shown in blue. Naïve $(n=4)$ tissue is shown in c. Qualitative analysis shows that scrambled ds-tat-treated tissue had elevated 3NT immunolabeling in comparison to naïve tissue, primarily in gray matter (d). This elevation grew through 7 days and was lessened at 28 days. Treatment with gp91ds-tat appeared to reduce this immunolabeling at all time points. Bar $=100 \mu \mathrm{m} .{ }^{*} p<0.05$ vs naïve; $+p<0.05$ vs scrambled; one-way ANOVA with Tukey's post-test. Bars represent mean \pm SEM 
scrambled ds-tat groups at any time point, although a trend toward significance was seen at all time points, with slight reductions in $3 \mathrm{NT}$ quantity in the white matter.

Finally, to determine whether NOX2 inhibition may interfere with NOX2 activity, phosphorylation of the NOX2 component $\mathrm{p} 47^{\mathrm{PHOX}}$ was assessed. Gp91ds-tat interferes with the activation of the NOX2 enzyme by blocking binding of phosphorylated $\mathrm{p} 47^{\mathrm{PHOX}}$ with the gp91 ${ }^{\text {PHOX }}$ subunit [6]. The expression of this enzyme is dependent on a feed-forward cycle, wherein ROS production by NOX2 can activate downstream signal transduction pathways that increase NOX2 component expression, including $\mathrm{p} 47^{\mathrm{PHOX}}$. Therefore, expression of phosphorylated $\mathrm{p} 47^{\mathrm{PHOX}}$ was assessed using western blotting (Fig. 3b). Phosphorylation of $\mathrm{p} 47^{\mathrm{PHOX}}$ was markedly elevated at $24 \mathrm{~h}$ post-injury, with no significant difference between groups. However, by 7 days post-injury, phosphorylation of $\mathrm{p} 47^{\mathrm{PHOX}}$ was significantly reduced in the gp91ds-tat-treated group, suggesting that activity of this enzyme was reduced at this time point, despite the lack of acute reduction (Fig. 3a).

\section{NOX2 inhibition reduces inflammatory cell populations in} the injured spinal cord

In order to determine the mechanism behind the improved functional outcome and determine the influence of reduced NOX2 activity and oxidative stress, flow cytometry was used to assess the percentages of macrophage, microglia, lymphocyte, and neutrophil populations at $24 \mathrm{~h}$ and 7 days post-injury. At $24 \mathrm{~h}$ post-injury, gp91ds-tat treatment was found to significantly reduce the neutrophil population $\left(\mathrm{CD} 45^{+} / \mathrm{GR}-1^{+}\right.$; Fig. 4b). By 7 days, macrophage/microglia $\left(\mathrm{CD} 45^{+} / \mathrm{CD} 1 \mathrm{~b}^{+} / \mathrm{GR}-1^{-}\right.$; Fig. 5b) was significantly reduced by gp91ds-tat administration. Neutrophils had a trend toward lower expression, although no significance was found (Fig. 5d). Further separation of the macrophage/microglia population by dividing this group into CD $45^{\text {high }}$ (macrophage) and CD $45^{\text {low }}$ (microglia) expression groups demonstrated that at 7 days post-injury, there was a greater proportion of microglia than macrophages in the injured spinal cord, and that this population demonstrated a greater response to gp91ds-tat treatment (Fig. 6b). No significant difference in T cell population $\left(\mathrm{CD}^{+} 5^{+} / \mathrm{CD}^{+}\right.$; Fig. 5f) was noted between treatment groups.

We next confirmed these flow cytometry results using immunohistochemistry. Immunostaining demonstrated a marked elevation in Iba1 staining in scrambled-tat-treated tissue from $24 \mathrm{~h}$ to 7 days post-injury (Fig. $7 \mathrm{a}-\mathrm{d}$ ). In tissue that had received gp91ds-tat administration, this elevation was not observed, and Iba1 staining was relatively low throughout the study.

NOX2 inhibition shifts microglia/macrophage polarization As gp91ds-tat was found to shift the inflammatory response, it was unclear if this had any effect on microglia/macrophage polarization. Previous studies have shown that NOX2 inhibition can alter polarization states [5]. We therefore assessed polarization markers in the spinal cord using flow cytometry, immunohistochemistry, and western blotting.

At 7 days post-injury, the M2 marker CD206 showed a significant increase with gp91ds-tat treatment compared to scrambled ds-tat, using both flow cytometry (Fig. 8) and western blotting (Fig. 9a).

Western blot data from $24 \mathrm{~h}$ post-injury revealed that expression of the M1 marker CD86 was significantly reduced in the gp91ds-tat group in comparison to the scrambled ds-tat group (Fig. 9b). However, expression of this protein then returned to levels demonstrated in the scrambled ds-tat-treated group by 7 and 28 days

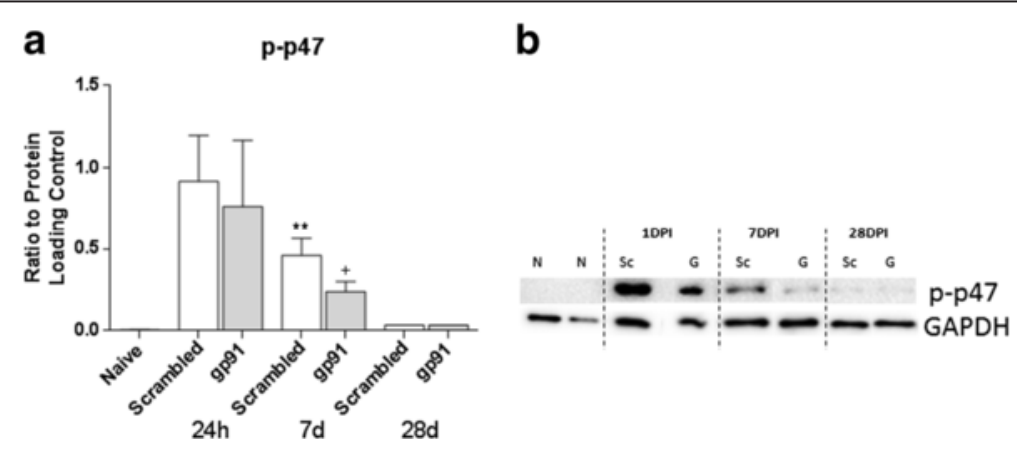

Fig. 3 Acute inhibition of NOX2 using gp91ds-tat reduces p47 ${ }^{\text {PHOX }}$ expression and/or phosphorylation. Phosphorylated p47 $7^{\text {PHOX }}$ (p-p47) and GAPDH were evaluated at $24 \mathrm{~h}, 7$ days, and 28 days post-injury in naïve $(N ; n=8)$, scrambled ds-tat- $(s ; n=4 / 24 \mathrm{~h} ; 6 / 7$ days; $3 / 28$ days), and gp91 ds-tat-treated samples (G; $n=4 / 24 \mathrm{~h} ; 5 / 7$ days; $3 / 28$ days). Bands were observed at 47 and $38 \mathrm{kDa}$, respectively, in a representative western blot (a). Pixel densitometry

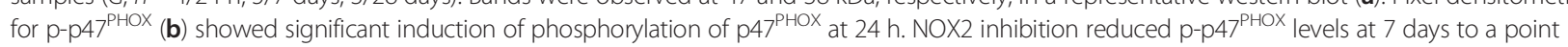
that was not significantly greater than sham. ${ }^{*} p<0.01$ vs naïve. $+p<0.01$ vs scrambled; one-way ANOVA with Tukey's post-test. Bars represent mean \pm SEM 


\section{a Scrambled ds-tat}

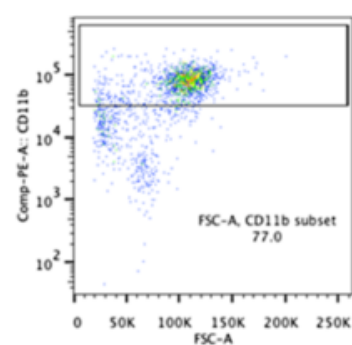

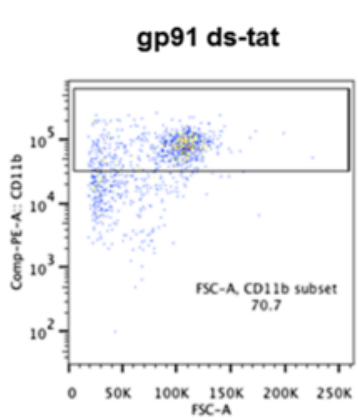

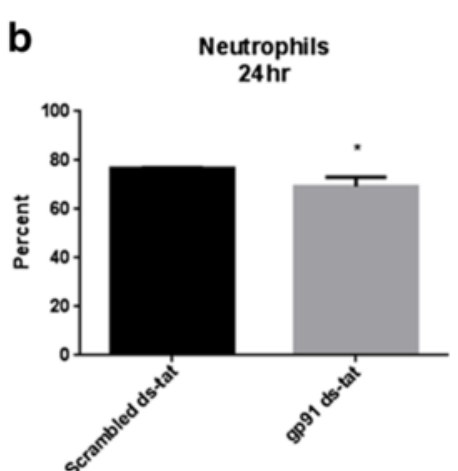

Fig. 4 Acute inhibition of NOX2 reduces neutrophil populations by $24 \mathrm{~h}$ post-injury. Neutrophils were characterized as alive, $\mathrm{CD} 45^{+} / \mathrm{CD} 11 \mathrm{~b}^{+} / \mathrm{GR}-1^{+}$ population (a). b Quantitation shows that gp91ds-tat treatment significantly reduced this population. ${ }^{*} p<0.05$ vs scrambled ds-tat, Student's $t$ test. Bars represent mean \pm SEM

\section{a Isotype Control}

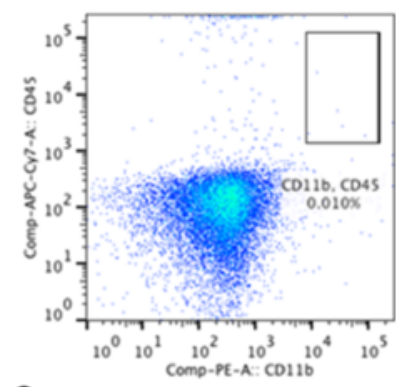

C

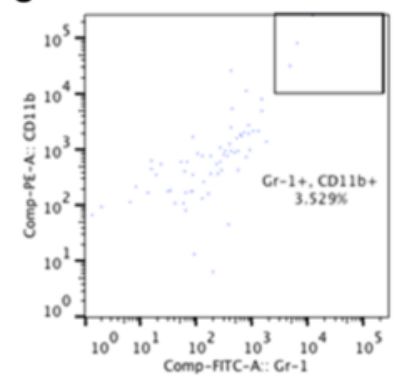

e

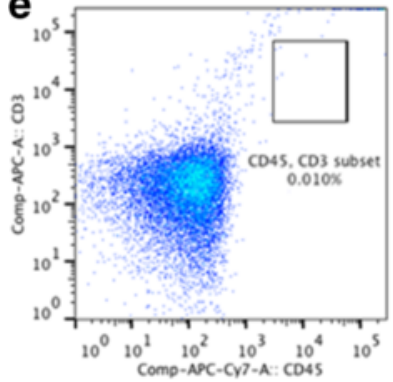

Scrambled ds-tat
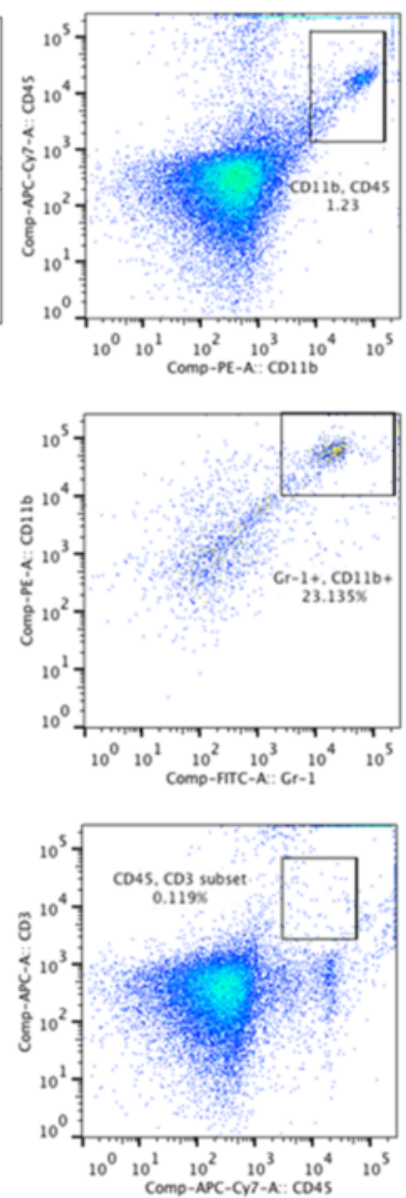
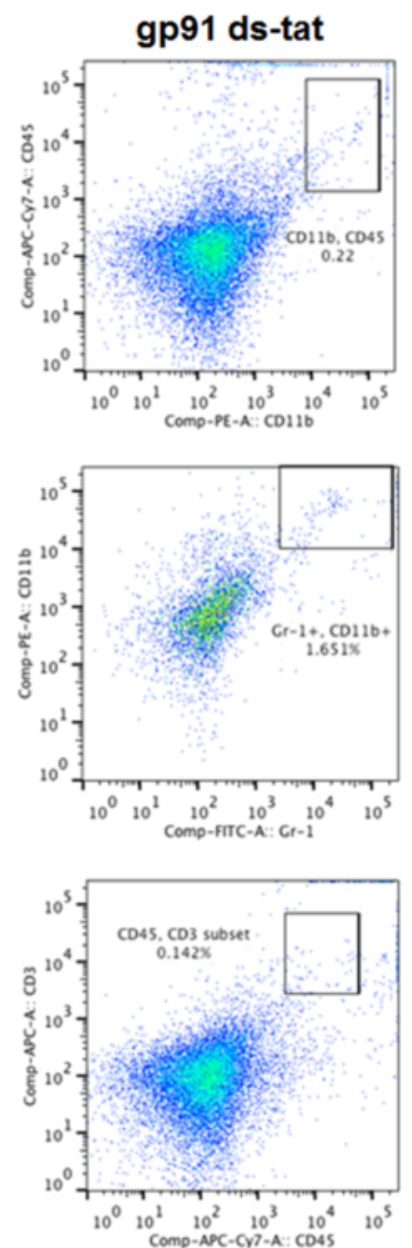

b

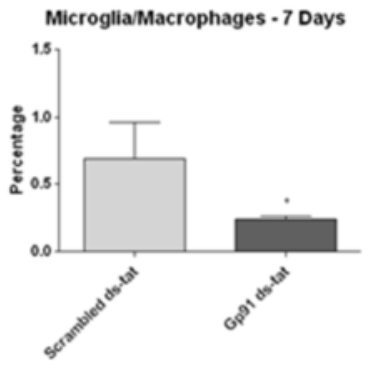

d
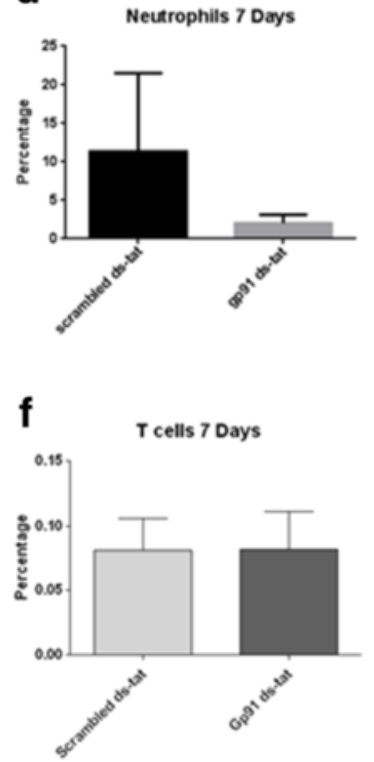

Fig. 5 Acute NOX2 inhibition reduces inflammatory response by 7 days post-injury. All listed cell types were under a live CD45+ gate. Microglia/ macrophage were further isolated as $\mathrm{CD}_{11} \mathrm{~b}^{+} \mathrm{Gr}-1^{-}$(a), neutrophils as $\mathrm{CD} 11 \mathrm{~b}^{+} \mathrm{Gr}-1^{+}(\mathbf{c})$, and $\mathrm{T}$ cells as $\mathrm{CD}^{+}(\mathbf{e})$. The first column in each row indicates an isotype control. Quantitation shows that gp91ds-tat treatment significantly reduced the macrophage/microglia (b) and neutrophil (d) populations but had no significant effect on the T cell population (f). ${ }^{*} p<0.05$ vs scrambled ds-tat, Student's $t$ test. Bars represent mean \pm SEM 

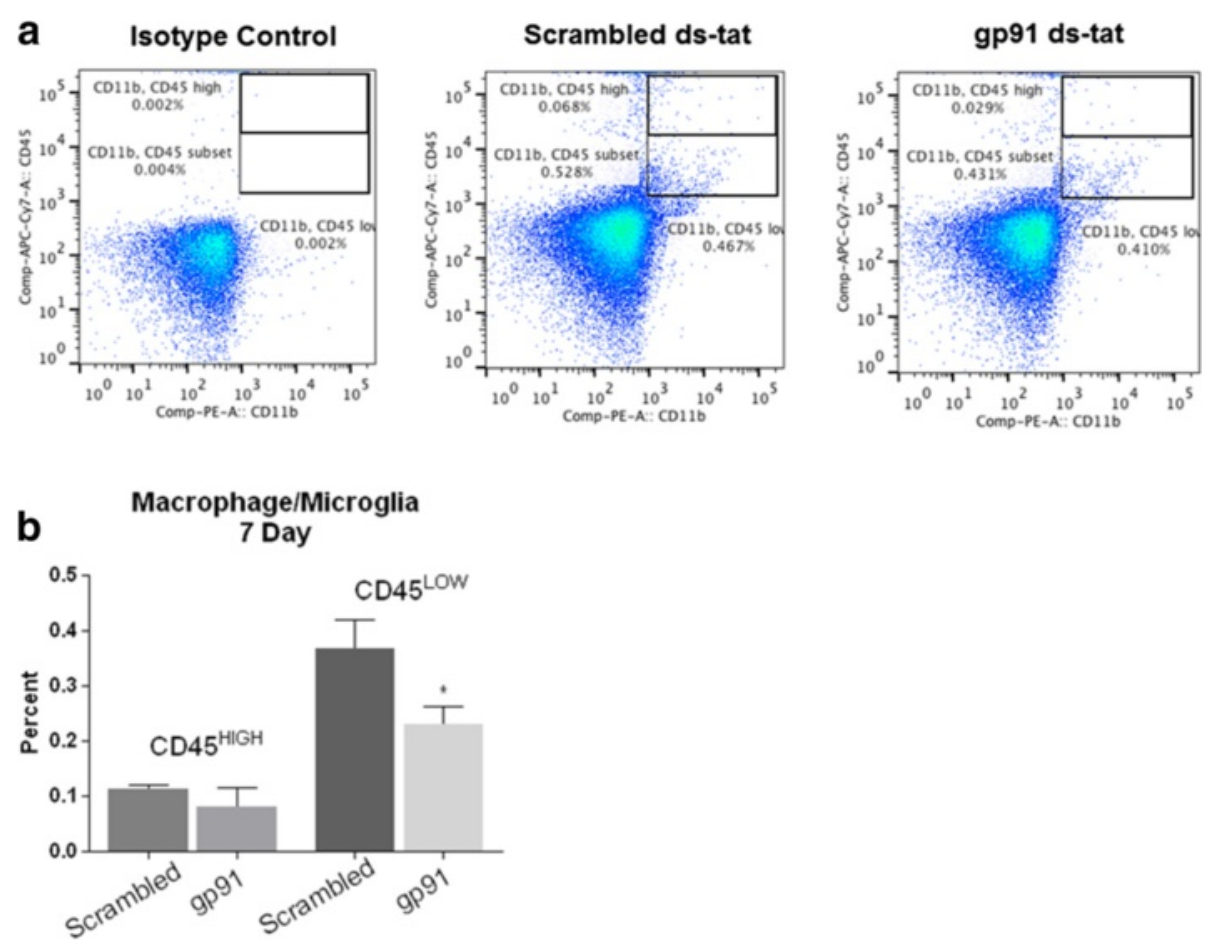

Fig. 6 Acute NOX2 inhibition specifically targets microglia. Flow cytometry was used to analyze macrophage and microglia populations in the injured region. Live cells were first gated on and further differentiated based on CD11 b and CD45 ${ }^{+}$. CD45 high, macrophages, and CD45 low, microglia, were arbitrarily chosen by a researcher blinded to study (a). Quantitation demonstrated no significant difference in macrophages at 7 days post-injury between scrambled ds-tat- and gp91ds-tat-treated tissue. Microglia, on the other hand, showed a significant decrease in tissue treated with gp91ds-tat compared to scrambled ds-tat (b). ${ }^{*} p<0.05$ vs scrambled ds-tat, Student's $t$ test. Bars represent mean \pm SEM

post-injury. An additional M1 marker, iNOS, demonstrated significantly reduced expression at 7 days postinjury, with no significant difference between groups at $24 \mathrm{~h}$ and 28 days (Fig. 9c).

\section{Discussion}

We now show that a single acute central administration of the NOX2 inhibitor gp91ds-tat led to significant improvements in a number of measures of recovery after moderate SCI. Recovery was seen on both the functional and cellular levels, including increases in BMS score and subscore, reductions in measures of oxidative stress, and reduced inflammatory cell presence through 7 days postinjury. Despite the observation that this single acute treatment did not induce inflammatory effects beyond 7 days, functional improvement continued through at least 28 days, indicating the importance of acute and subacute interventions.

Although the NADPH complex enzyme group encompasses several different NOX and DUOX complexes, our use of gp91ds-tat was specific to the NOX2 isoform. This peptide contains a sequence that mimics gp91 ${ }^{\mathrm{PHOX}}$ and binds to phosphorylated $\mathrm{p} 47^{\mathrm{PHOX}}$ [15]. This peptide then blocks ROS production specifically by the NOX2 isoform, having no effect on NOX1 or 4 [16]. In vitro, gp91ds-tat has been shown to block the assembly of the NOX2 enzyme [17]. In vivo, gp91ds-tat reduced neuronal death in a brain injury model when administered as a single pre-treatment dose [13].

A number of NOX inhibitors have been used in the past. These include apocynin, which interferes with the assembly of the active NOX enzyme [18], and diphenyleneiodonium (DPI), which directly blocks the catalytic activity of NOX (and other flavoprotein-containing enzymes) [19]. Apocynin has been shown to reduce oxidative stress in the central nervous system during conditions including sepsis [20] and brain injury [13, 21], providing significant neuroprotection. More importantly, apocynin administration following SCI in rats significantly reduced a number of inflammatory and oxidative stress markers, including neutrophil invasion, nitrotyrosine production, and pro-inflammatory cytokine expression [22]. Further, DPI administration reduced oligodendrocyte and oligodendrocyte precursor death in lipopolysaccharide induced inflammation models [23]. More importantly, we have shown that DPI administration following SCI reduces lesion volume and post-injury inflammation [9]. However, DPI and apocynin have been found to have numerous non-specific effects and can act on enzymes beyond the NOX family that contain flavoproteins [24]. 

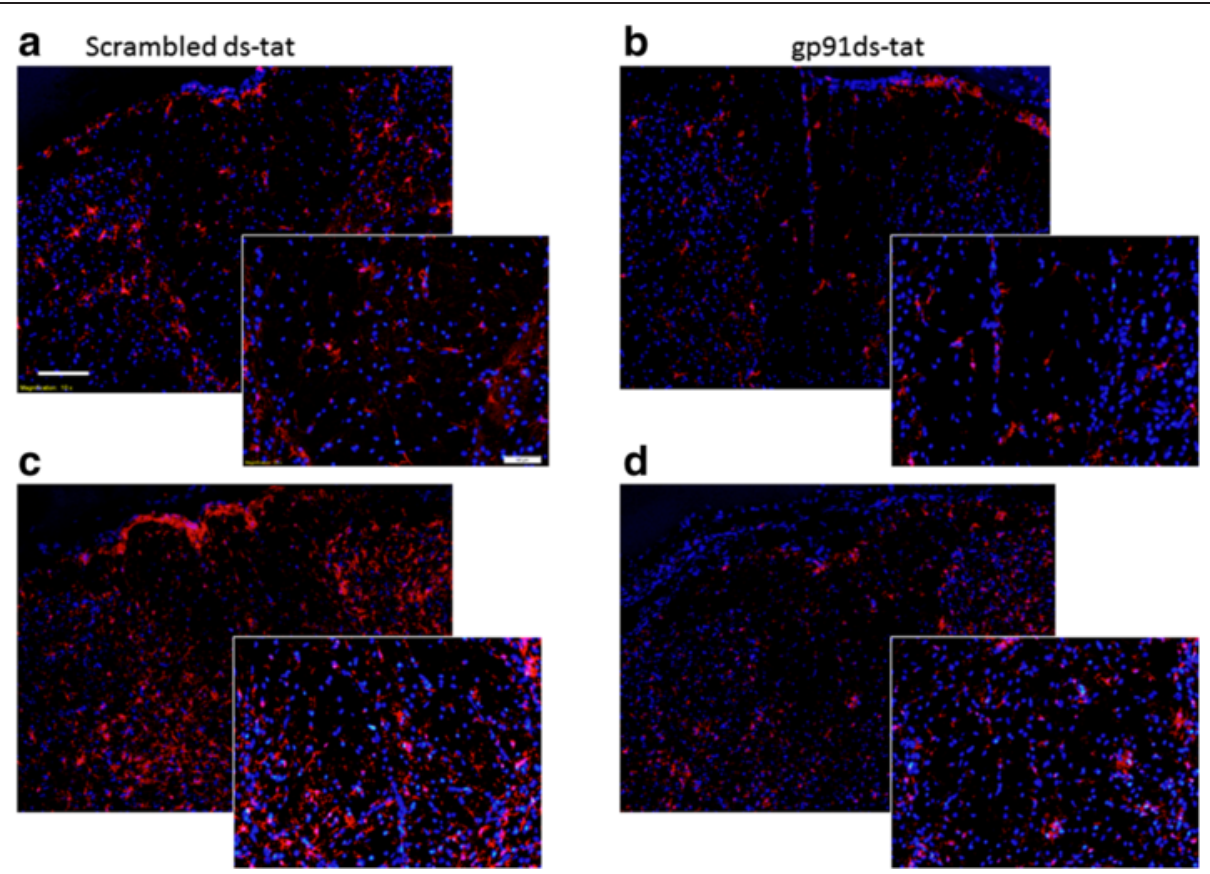

Fig. 7 Acute NOX2 inhibition reduces microglia/macrophage presence in injured spinal cord tissue. Gp91ds-tat- and scrambled ds-tat-treated tissue at $24 \mathrm{~h}$ ( $\mathbf{a}, \mathbf{b} ; n=4 /$ group) and 7 days (c, $\mathbf{d} ; n=3$ gp91ds-tat, 4 scrambled ds-tat) post-injury was stained for the pan microglia/macrophage marker Iba1 (red). DAPI nuclear stain is shown in blue. At $24 \mathrm{~h}$ post-injury, the scrambled ds-tat-treated tissue (a) have a greater number of activated microglia compared to gp91ds-tat-treated tissue as seen in $\times 10$ magnification (b). The activated morphology includes a more amoeboid body with retracted dendrites (which can be better observed in the higher magnification inset image ( $\times 20)$ ). At the same time point, the microglia present in gp91ds-tat tissue have long processes and smaller bodies. This difference is greater at the 7-day time point when microglia are at their peak (c, $\mathbf{d}$ ). The gp91 ds-tat-treated microglia at 7 days $(\times 20)$ are activated, but the number are still downregulated compared to those treated with scrambled-tat. Lower magnification and high-magnification images shown; bar ( $\times 20)=50 \mu \mathrm{m}$; bar ( $\times 10)=100 \mu \mathrm{m}$

While picomolar concentrations have been shown to reduce these flavoprotein effects [25], these drugs are still not specific for any particular NOX isoform. In contrast, we now show that specific and acute inhibition of the NOX2 isoform can reduce inflammation in both the acute and subacute periods, leading to long-term functional improvement.
The functional improvement observed was measured using both the BMS and the BMS subscore, suggesting improvements in both gross and fine motor skills. These improvements first became significant at 14 days postinjury, although a trend was observed as early as 7 days, which is the time point at which significant differences in several markers of oxidative stress and inflammation

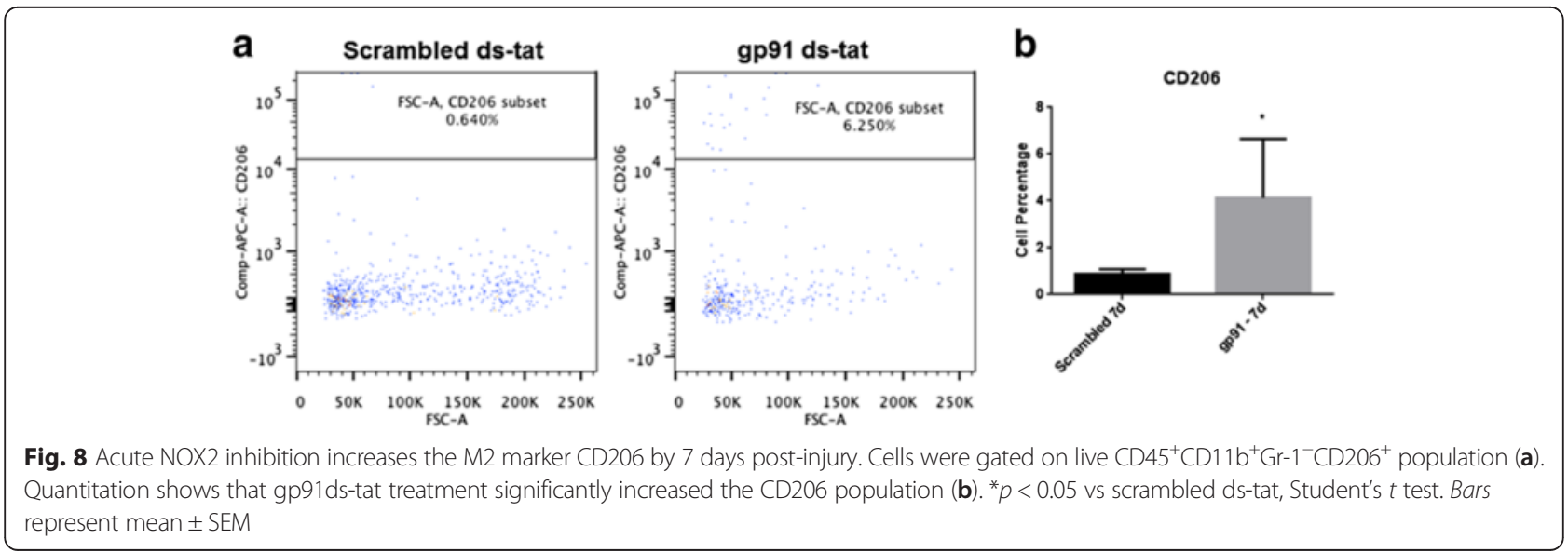


a

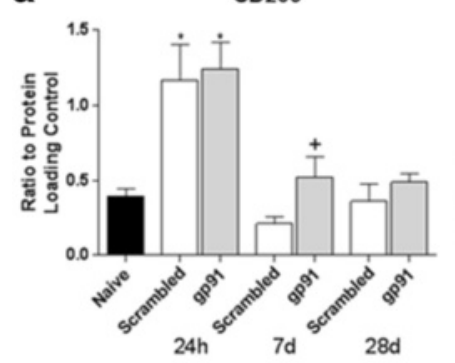

b
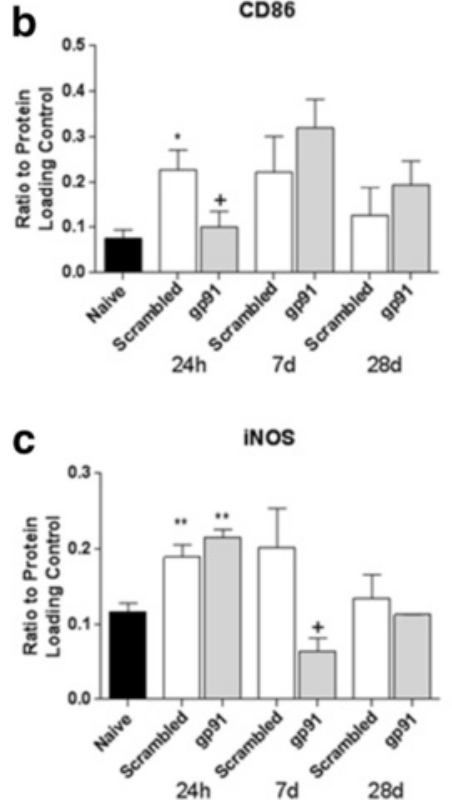
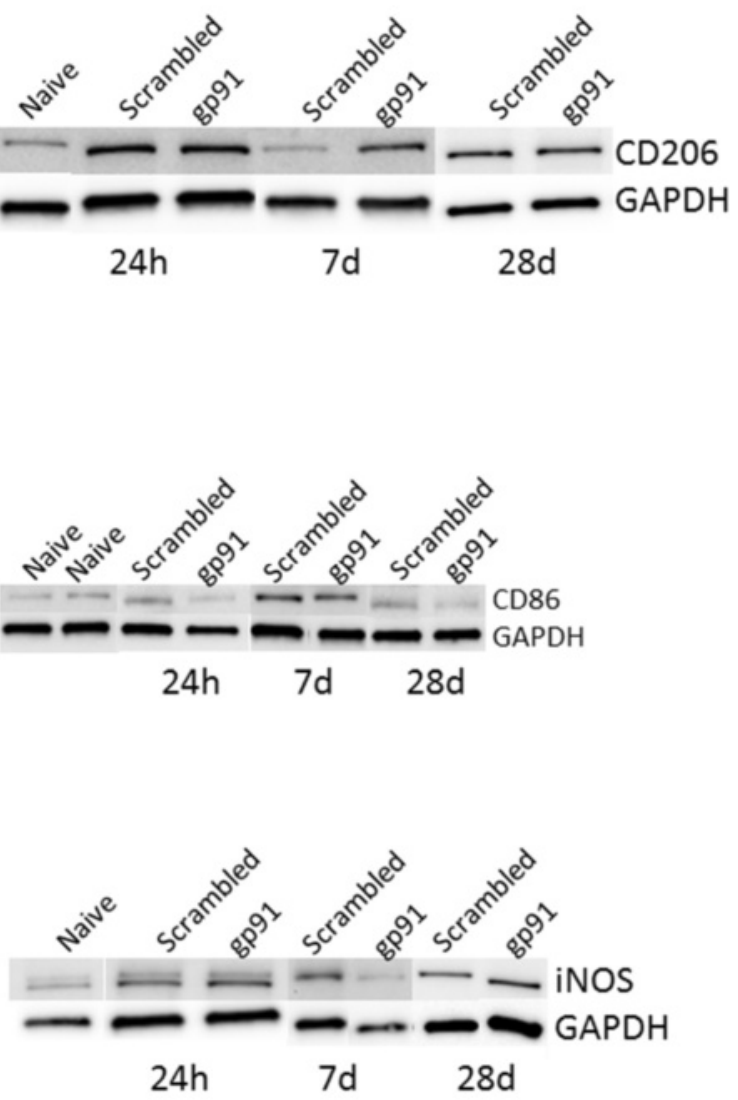

$24 \mathrm{~h}$

$7 \mathrm{~d}$ $28 \mathrm{~d}$

Fig. 9 Acute inhibition of NOX2 using gp91ds-tat alters microglia/macrophage polarization marker expression. Protein samples $(25 \mu \mathrm{g})$ were probed for CD206, iNOS, CD86, and GAPDH at 24 h, 7 days, and 28 days post-injury in naïve $(n=8)$, scrambled ds-tat- $(n=4 / 24$ h; $6 / 7$ days; $3 / 28$ days), and gp91ds-tat-treated samples ( $n=4 / 24$ h; 5/7 days; $3 / 28$ days). Pixel densitometry for CD206 (a), CD86 (b)), and iNOS (c) showed significant alteration with gp91ds-tat treatment in comparison to scrambled ds-tat treatment. The M2 marker CD206 was elevated in both groups at $24 \mathrm{~h}$ in comparison to naïve, but remained elevated only in the gp91ds-tat-treated group at 7 days post-injury, before returning to baseline levels by 28 days. In contrast, at $24 \mathrm{~h}$, the inhibition of NOX2 significantly reduced the expression of the M1 marker CD86, although by 7 and 28 days, no significant difference was observed between groups. Finally, a second M1 marker, iNOS, was elevated in both groups at $24 \mathrm{~h}$ post-injury, and gp91ds-tat treatment only prevented this induction by 7 days post-injury. ${ }^{*} p<0.05$ vs naïv; $+p<0.05$ vs scrambled; one-way ANOVA with Tukey's post-test. Bars represent mean \pm SEM

were observed. Despite the fact that no significant difference in oxidative stress marker or inflammation was observed at 28 days post-injury, functional improvement continued through 28 days, suggesting that acute inhibition of NOX2 was sufficient to result in sustained functional changes. However, it is important to note that by 21-28 days post-injury, the significance in the BMS subscore between the gp91ds-tat- and scrambled ds-tattreated groups was lost, suggesting that sustained or even delayed administration of treatment may have further benefits.

A number of studies have shown that NOX isoforms are upregulated after central nervous system injury, including brain injury $[12,13]$ and SCI [7-11], and may contribute to post-injury oxidative stress. This study adds to that literature, demonstrating elevated protein carbonylation and nitrosylation in combination with elevated expression and/or phosphorylation of $\mathrm{p} 47^{\mathrm{PHOX}}$. Phosphorylation of the $\mathrm{p} 47^{\text {PHOX }}$ subcomponent results in unmasking of PHOX-binding domains in the protein, allowing for assembly and activation of the enzyme [26]. Interestingly, we found the greatest elevation in protein expression of the NOX2 component at $24 \mathrm{~h}$ after injury, with expression and phosphorylation reduced by 7 days and returning to baseline levels by 28 days. This is in contrast to our previous work, wherein gene and protein expression of NOX2 components gp91 ${ }^{\mathrm{PHOX}}$ and $\mathrm{p} 22^{\mathrm{PHOX}}$ as well as NOX activity were elevated for months after injury [9]. However, the extended expression profile was determined in a rat model of SCI; it is likely that the mouse model used in the 
current study demonstrates a different NOX expression and activity profile. Future research will continue to explore these differences. Regardless, the evidence indicates that NOX2 is active for at least 7 days post-injury, suggesting that a delayed treatment approach may be efficacious in this model and others.

Concurrently or following this alteration in oxidative stress status, we observed a significant reduction in post-injury inflammation, including acute and subacute reductions in neutrophil invasion and subacute reductions in macrophage/microglial populations with gp91dstat administration. Interestingly, microglial populations, defined by low expression of the CD45 marker, were most affected by gp91ds-tat administration. This is likely due to the local rather than systemic administration of the NOX2 inhibitor. Systemic administration of apocynin, a non-specific NOX inhibitor, has been shown to similarly reduce inflammatory responses, but without specific effects on microglia [22]. Further, this study demonstrated that apocynin administration reduced adhesion molecule expression, which may provide an explanation for the reduction in neutrophil invasion observed with local spinal cord injection of gp91ds-tat. In addition, while analysis with both flow cytometry and immunohistochemistry demonstrated no significant difference in microglia/macrophage presence at $24 \mathrm{~h}$, qualitative analysis suggested a morphological change, with fewer "activated" macrophages/microglia at this time point in gp91ds-tattreated tissue. Activated microglia/macrophages are known to demonstrate an amoeboid structure with retracted dendrites, which was seen in tissue that received the scrambled ds-tat control treatment; gp91dstat-treated tissue demonstrated a qualitatively greater presence of ramified cells, with small cell bodies and long thin processes [27].

To further explore the phenotype of the affected macrophages and microglia, cellular polarization was investigated. In 2009, Kigerl et al. [4] demonstrated that there is a balance of M1 and M2 markers acutely after injury that is followed by an increase in the M1:M2 ratio in the subacute period. Our data support these findings, with elevated M1 and M2 marker expression at acute time points and a shift toward M1 dominance by 7 days post-injury. Gp91ds-tat administration appears to limit this shift, however, reducing the decline of the M2 polarization marker at 7 days post-injury (Figs. 8 and 9a) and reducing M1 markers at 1 or 7 days post-injury (Fig. 9b, c). The influence of NOX2 inhibition on M1 marker expression was somewhat variable, with acute effects observed in the CD86 marker and subacute effects in the iNOS marker; this may reflect a variability in polarization phenotypes over time. Future research is needed to more precisely identify the influence of NOX inhibition on polarization. Further, it should be noted that M1 and M2 marker expression in the gp91ds-tattreated group had returned to scrambled ds-tat-treated group levels by 28 days post-injury, suggesting that a single acute administration is insufficient to retain a long-lasting alteration in polarization.

Despite this, we, and others, have now shown that acute alterations in microglial polarization and NOX activity can influence each other. Administration of the M2polarizing cytokine IL4 induced a reduction in NOX2 expression by $24 \mathrm{~h}$ [28]. Inhibition of NOX activity, via knockout of $\mathrm{p} 47^{\mathrm{PHOX}}$ and $\mathrm{gp} 91^{\mathrm{PHOX}}$ or administration of apocynin, on the other hand, significantly reduced M1 polarization and shifted microglia in LPS-treated brain tissue to an M2 polarization state [5]. This shift was accompanied by a reduction in pro-inflammatory cytokines and Iba1 immunolabeling. These results are supported by our current study and extended to the spinal cord trauma field, demonstrating that inhibition of the NOX2 isoform specifically reduces M1 polarization, increases M2 polarization, and reduces a number of proinflammatory markers.

In conclusion, these data demonstrate that a single acute NOX2-specific inhibition can have dramatic effects on oxidative stress, inflammation, and microglial polarization following SCI, and that these effects significantly improve post-injury functional recovery. NOX2, therefore, plays an integral role in post-injury inflammation, and specific activity on this enzyme can be targeted in order to help avoid non-target effects. Despite the marked alterations, we did observe a return to untreated injury levels in many of our outcome measures by 1 month post-injury, suggesting that work remains to elucidate the appropriate treatment approach in order for this therapy to be considered for further development and clinical use.

\section{Competing interests}

The authors declare that they have no competing interests.

\section{Authors' contributions}

GK completed all the surgeries and tissue analysis, including immunohistochemistry, western blot, and flow cytometry, and drafted the manuscript. SB carried out the antibody optimization for the immunohistochemistry and assisted with the western blotting and tissue preparation. KRB conceived of the study and participated in its design and coordination and helped draft the manuscript. All authors read and approved the final manuscript.

\section{Acknowledgements}

The authors thank Kateryna Lund, MS, Mouna Lagraoui, PhD, Natallia Mikhalkevich, MS, and Jonathan Godbout, PhD. for the assistance with the flow cytometry design and interpretation. This work was funded by the NINDS/NIH (grant number 1R01NS073667-01A1).

Received: 4 June 2015 Accepted: 3 September 2015

Published online: 17 September 2015

\section{References}

1. Fleming JC, Norenberg MD, Ramsay DA, Dekaban GA, Marcillo AE, Saenz $A D$, et al. The cellular inflammatory response in human spinal cords after injury. Brain. 2006;129:3249-69. 
2. Kroner A, Greenhalgh AD, Zarruk JG, Passos Dos Santos R, Gaestel M, David S. TNF and increased intracellular iron alter macrophage polarization to a detrimental M1 phenotype in the injured spinal cord. Neuron. 2014:83:1098-116.

3. Yao A, Liu F, Chen K, Tang L, Liu L, Zhang K, et al. Programmed death 1 deficiency induces the polarization of macrophages/microglia to the $M 1$ phenotype after spinal cord injury in mice. Neurotherapeutics. 2014;11:636-50.

4. Kigerl KA, Gensel JC, Ankeny DP, Alexander JK, Donnelly DJ, Popovich PG. Identification of two distinct macrophage subsets with divergent effects causing either neurotoxicity or regeneration in the injured mouse spinal cord. J Neurosci. 2009;29:13435-44.

5. Choi SH, Aid S, Kim HW, Jackson SH, Bosetti F. Inhibition of NADPH oxidase promotes alternative and anti-inflammatory microglial activation during neuroinflammation. J Neurochem. 2012;120:292-301.

6. Bedard K, Krause $\mathrm{KH}$. The NOX family of ROS-generating NADPH oxidases: physiology and pathophysiology. Physiol Rev. 2007:87:245-313.

7. Vaziri ND, Lee YS, Lin CY, Lin WW, Sindhu RK. NAD(P)H oxidase, superoxide dismutase, catalase, glutathione peroxidase and nitric oxide synthase expression in subacute spinal cord injury. Brain Res. 2004;995:76-83.

8. Byrnes KR, Garay J, Di Giovanni S, De Biase A, Knoblach SM, Hoffman EP, et al. Expression of two temporally distinct microglia-related gene clusters after spinal cord injury. Glia. 2006;53:420-33.

9. Byrnes KR, Washington PM, Knoblach SM, Hoffman E, Faden Al. Delayed inflammatory mRNA and protein expression after spinal cord injury. J Neuroinflammation. 2011:8:130.

10. Pajoohesh-Ganji A, Knoblach SM, Faden Al, Byrnes KR. Characterization of inflammatory gene expression and galectin-3 function after spinal cord injury in mice. Brain Res. 2012;1475:96-105.

11. Cooney SJ, Zhao Y, Byrnes KR. Characterization of the expression and inflammatory activity of NADPH oxidase after spinal cord injury. Free Radic Res. 2014;48:929-39.

12. Cooney SJ, Bermudez-Sabogal SL, Byrnes KR. Cellular and temporal expression of NADPH oxidase (NOX) isotypes after brain injury. J Neuroinflammation. 2013;10:155.

13. Zhang QG, Laird MD, Han D, Nguyen K, Scott E, Dong Y, et al. Critical role of NADPH oxidase in neuronal oxidative damage and microglia activation following traumatic brain injury. PLoS One. 2012;7:e34504.

14. Donnelly DJ, Gensel JC, Ankeny DP, van Rooijen N, Popovich PG. An efficient and reproducible method for quantifying macrophages in different experimental models of central nervous system pathology. J Neurosci Methods. 2009;181:36-44.

15. Kleniewska P, Piechota A, Skibska B, Goraca A. The NADPH oxidase family and its inhibitors. Arch Immunol Ther Exp (Warsz). 2012;60:277-94.

16. Csanyi G, Cifuentes-Pagano E, Al Ghouleh I, Ranayhossaini DJ, Egana L, Lopes LR, et al. Nox2 B-loop peptide, Nox2ds, specifically inhibits the NADPH oxidase Nox2. Free Radic Biol Med. 2011;51:1116-25.

17. Jackson EK, Gillespie DG, Zhu C, Ren J, Zacharia LC, Mi Z. Alpha2-adrenoceptors enhance angiotensin I-induced renal vasoconstriction: role for NADPH oxidase and RhoA. Hypertension. 2008;51:719-26.

18. Otto A, Fontaine D, Fontaine J, Berkenboom G. Rosuvastatin treatment protects against nitrate-induced oxidative stress. J Cardiovasc Pharmacol. 2005;46:177-84.

19. Doussiere J, Bouzidi F, Poinas A, Gaillard J, Vignais PV. Kinetic study of the activation of the neutrophil NADPH oxidase by arachidonic acid. Antagonistic effects of arachidonic acid and phenylarsine oxide. Biochemistry. 1999;38:16394-406.

20. Hernandes MS, D'Avila JC, Trevelin SC, Reis PA, Kinjo ER, Lopes LR, et al. The role of Nox2-derived ROS in the development of cognitive impairment after sepsis. J Neuroinflammation. 2014;11:36.

21. Choi BY, Jang BG, Kim JH, Lee BE, Sohn M, Song HK, et al. Prevention of traumatic brain injury-induced neuronal death by inhibition of NADPH oxidase activation. Brain Res. 2012;1481:49-58.

22. Impellizzeri D, Mazzon E, Esposito E, Paterniti I, Bramanti P, Cuzzocrea S. Effect of Apocynin, an inhibitor of NADPH oxidase, in the inflammatory process induced by an experimental model of spinal cord injury. Free Radic Res. 2011:45:221-36.

23. He YF, Chen HJ, Qian LH, He LF, Buzby JS. Diphenyleneiodonium protects preoligodendrocytes against endotoxin-activated microglial NADPH oxidase-generated peroxynitrite in a neonatal rat model of periventricular leukomalacia. Brain Res. 2013;1492:108-21.
24. Cheret C, Gervais A, Lelli A, Colin C, Amar L, Ravassard P, et al. Neurotoxic activation of microglia is promoted by a nox1-dependent NADPH oxidase. J Neurosci. 2008;28:12039-51

25. Wang Q, Chu CH, Oyarzabal E, Jiang L, Chen SH, Wilson B, et al. Subpicomolar diphenyleneiodonium inhibits microglial NADPH oxidase with high specificity and shows great potential as a therapeutic agent for neurodegenerative diseases. Glia. 2014;62:2034-43.

26. Groemping Y, Lapouge K, Smerdon SJ, Rittinger K. Molecular basis of phosphorylation-induced activation of the NADPH oxidase. Cell. 2003;113:343-55.

27. Byrnes KR, Loane DJ, Stoica BA, Zhang J, Faden Al. Delayed mGluR5 activation limits neuroinflammation and neurodegeneration after traumatic brain injury. J Neuroinflammation. 2012:9:43.

28. Savchenko VL. Regulation of NADPH oxidase gene expression with PKA and cytokine IL-4 in neurons and microglia. Neurotox Res. 2013;23:201-13.

\section{Submit your next manuscript to BioMed Central and take full advantage of:}

- Convenient online submission

- Thorough peer review

- No space constraints or color figure charges

- Immediate publication on acceptance

- Inclusion in PubMed, CAS, Scopus and Google Scholar

- Research which is freely available for redistribution 